\title{
GENERALIZATION OF H. MINC AND L. SATHRE'S INEQUALITY
}

\author{
FENG QI AND QIU-MING LUO
}

Abstract. An inequality of H. Minc and L. Sathre (Proc. Edinburgh Math. Soc. 14(1964/65), 41-46) is generalized as follows: Let $n$ and $m$ be natural numbers, $k$ a nonnegative integer, then we have

$$
\frac{n+k}{n+m+k}<\frac{\sqrt[n]{(n+k) ! / k !}}{\sqrt[n+m]{(n+m+k) ! / k !}}<1 .
$$

From this, some corollaries are deduced. At last, an open problem is proposed.

It is known that, for $n \in \mathbb{N}$, the following inequalities were given in [3];

$$
\frac{n}{n+1}<\frac{\sqrt[n]{n !}}{\sqrt[n+1]{(n+1) !}}<1
$$

in [1], the left inequality in (1) was refined by

$$
\frac{n}{n+1}<\left(\frac{1}{n} \sum_{i=1}^{n} i^{r} / \frac{1}{n+1} \sum_{i=1}^{n+1} i^{r}\right)^{1 / r}<\frac{\sqrt[n]{n !}}{\sqrt[n+1]{(n+1) !}}
$$

for all positive real numbers $r$. Both bounds are best possible.

In this article, using analytic method, we obtain

Theorem. Let $n$ and $m$ be natural numbers, $k$ a nonnegative integer. Then we have

$$
\frac{n+k}{n+m+k}<\frac{\sqrt[n]{(n+k) ! / k !}}{\sqrt[n+m]{(n+m+k) ! / k !}}<1
$$

Proof. The upper bound is obtained immediately from

$$
\frac{\sqrt[n]{(n+k) ! / k !}}{\sqrt[n+m]{(n+m+k) ! / k !}}=\left[\left(\prod_{i=k+1}^{n+k} i\right)^{m} /\left(\prod_{i=n+k+1}^{n+m+k} i\right)^{n}\right]^{1 / n(n+m)}<1 .
$$

Received May 10, 1999.

2000 Mathematics Subject Classification. Primary 26D15.

Key words and phrases. Generalization, H. Minc and L. Sathre's inequality.

The first author was supported in part by NSF of Henan Province, SF of the Education Committee of Henan Province (1999110004) and Doctor Fund of Jiaozuo Institute of Technology, The People's Republic of China. 
The left inequality in (3) can be rearranged as

$$
\frac{n+k}{\sqrt[n]{(n+k) ! / k !}}<\frac{n+m+k}{\sqrt[n+m]{(n+m+k) ! / k !}}
$$

this is equivalent to

$$
\frac{n+k}{\sqrt[n]{(n+k) ! / k !}}<\frac{n+k+1}{\sqrt[n+1]{(n+k+1) ! / k !}}
$$

When $k=0$, inequality (4) follows from the left inequality in (1). When $k \geq 1$, the inequality (4) can be rewritten as

$$
\left[\frac{(n+k) !}{k !}\right]^{1 / n}>\frac{(n+k)^{n+1}}{(n+k+1)^{n}} .
$$

In $[4$, p. 184], the following inequalities were given for $n \in \mathbb{N}$.

$$
\sqrt{2 \pi n}\left(\frac{n}{e}\right)^{n}<n !<\sqrt{2 \pi n}\left(\frac{n}{e}\right)^{n} \exp \frac{1}{12 n} .
$$

By substituting the inequalities in (6) into the left term of inequality (5), we see that it is sufficient to prove

$$
\left[\sqrt{2 \pi(n+k)}\left(\frac{n+k}{e}\right)^{n+k}\right]^{1 / n}>\frac{(n+k)^{n+1}}{(n+k+1)^{n}}\left[\sqrt{2 \pi k}\left(\frac{k}{e}\right)^{k} \exp \frac{1}{12 k}\right]^{1 / n}
$$

Simplifying (7) directly and standard arguments leads to

$$
n \ln \left(1+\frac{1}{n+k}\right)+\frac{2 k+1}{2 n} \ln \left(1+\frac{n}{k}\right)-\frac{1}{12 k n}-1>0 .
$$

In [2, pp.367-368], [4, pp.273-274] and [8], we have for $t>0$

$$
\ln \left(1+\frac{1}{t}\right)>\frac{2}{2 t+1}
$$

Thus, to get inequality (8), it suffices to show

$$
\frac{2 n}{2(n+k)+1}+\frac{2 k+1}{2 n} \cdot \frac{2 n}{2 k+n}-\frac{1}{12 k n}-1>0 .
$$

But this is equivalent to

$$
2\left(12 k^{2}-1\right) n^{2}+(12 k n-1) n+4(6 n-1) k^{2}+2(3 n-1) k>0 .
$$

The proof is complete. 
Corollary 1. For any given nonnegative integer $k$, the sequences

$$
\begin{aligned}
& \sqrt[n]{(n+k) ! / k !} \\
& \frac{n+k}{\sqrt[n]{(n+k) ! / k !}} \\
& \frac{(n+k) \sqrt[n+1]{(n+k+1) ! / k !}}{\sqrt[n]{(n+k) ! / k !}} \\
& \frac{(n+k+1) \sqrt[n]{(n+k) ! / k !}}{\sqrt[n+1]{(n+k+1) ! / k !}}
\end{aligned}
$$

are strictly increasing with respect to $n \in \mathbb{N}$.

Corollary 2. For any given $n \in \mathbb{N}$, the sequences

$$
\sqrt[n]{(n+k) ! / k !}, \quad \frac{(n+k) \sqrt[n+1]{(n+k+1) ! / k !}}{\sqrt[n]{(n+k) ! / k !}}, \quad \frac{(n+k+1) \sqrt[n]{(n+k) ! / k !}}{\sqrt[n+1]{(n+k+1) ! / k !}}
$$

are strictly increasing with respect to the nonnegative integers $k$.

Remark. Recently, the first author in [5] and [7], among other things, generalized the left inequality in (2) in new directions and got that, if $n$ and $m$ are natural numbers, $k$ is a nonnegative integer, then

$$
\frac{n+k}{n+m+k}<\left(\frac{1}{n} \sum_{i=k+1}^{n+k} i^{r} / \frac{1}{n+m} \sum_{i=k+1}^{n+m+k} i^{r}\right)^{1 / r}
$$

where $r$ is any given positive real number. The lower bound is best possible.

In [6], the first author further presented that, let $n$ and $m$ be natural numbers, suppose $a=\left(a_{1}, a_{2}, \ldots\right)$ is a positive and increasing sequence satisfying

$$
\begin{aligned}
a_{k+1}^{2} & \geq a_{k} a_{k+2}, \\
\frac{a_{k+1}-a_{k}}{a_{k+1}^{2}-a_{k} a_{k+2}} & \geq \max \left\{\frac{k+1}{a_{k+1}}, \frac{k+2}{a_{k+2}}\right\}
\end{aligned}
$$

for $k \in \mathbb{N}$, then the inequality

$$
\frac{a_{n}}{a_{n+m}} \leq\left(\frac{1}{n} \sum_{i=1}^{n} a_{i}^{r} / \frac{1}{n+m} \sum_{i=1}^{n+m} a_{i}^{r}\right)^{1 / r}
$$

holds for any given positive real number $r \in \mathbb{R}$. The lower bound of (12) is best possible.

Using L'Hospital principle yields

$$
\lim _{r \rightarrow 0}\left(\frac{1}{n} \sum_{i=k+1}^{n+k} i^{r} / \frac{1}{n+m} \sum_{i=k+1}^{n+m+k} i^{r}\right)^{1 / r}=\frac{\sqrt[n]{(n+k) ! / k !}}{\sqrt[n+m]{(n+m+k) ! / k !}}
$$

thus, we propose the following 
Open Problem. Let $n$ and $m$ be natural numbers, $k$ a nonnegative integer. Then, for all real numbers $r>0$, we have

$$
\left(\frac{1}{n} \sum_{i=k+1}^{n+k} i^{r} / \frac{1}{n+m} \sum_{i=k+1}^{n+m+k} i^{r}\right)^{1 / r}<\frac{\sqrt[n]{(n+k) ! / k !}}{\sqrt[n+m]{(n+m+k) ! / k !}}
$$

The upper bound is best possible.

\section{References}

[1] H. Alzer, On an inequality of H. Minc and L. Sathre, J. Math. Anal. Appl., 179(1993), 396-402.

[2] Ji-Chang Kuang, Applied Inequalities, 2nd edition, Hunan Education Press, Changsha, China, 1993, Chinese.

[3] H. Minc and L. Sathre, Some inequalities involving $(r !)^{1 / r}$, Proc. Edinburgh Math. Soc., 14(1964/65), 41-46.

[4] D. S. Mitrinović, Analytic Inequalities, Springer-Verlag, New York/Heidelberg/Berlin, 1970.

[5] Feng Qi, An algebraic inequality, RGMIA Research Report Collection, 2(1999), 81-83. http://rgmia.vu.edu.au.

[6] Feng Qi and Lokenath Debnath, On a new generalization of H. Alzer's inequality, Intern. J. Math. \& Math. Sci., 25(2000), in the press. http://rgmia.vu.edu.au.

[7] Feng Qi, Generalization of H. Alzer's inequality, J. Math. Anal. Appl., (1999), in the press.

[8] Feng Qi and Chao-Ping Chen, Monotonicities of two sequences, Mathematics and Informatics Quarterly, 1999, to appear.

Department of Mathematics, Jiaozuo Institute of Technology, Jiaozuo City, Henan 454000, The People's Republic of China.

E-mail: qifeng@jzit.edu.cn

Department of Broadcast-Television Teaching, Jiaozuo University, Jiaozuo City, Henan 454151, The People's Republic of China. 